\title{
USING ULTRASONIC MEASUREMENTS AND A TWO-PHASE COMPOSITE MODEL TO ASSESS RADIATION DAMAGE IN REACTOR PRESSURE VESSEL STEELS
}

\author{
J. A. Wang \\ Computational Physics and Engineering Division \\ Oak Ridge National Laboratory" \\ P.O. Box 2008 \\ Oak Ridge, Tennessee 37831-6370
}

\author{
To be presented at \\ ASTM Symposium on Nontraditional \\ Methods of Sensing Stress, Strain, and \\ Damage in Material and Structures \\ Orlando, Florida \\ May 20, 1996
}

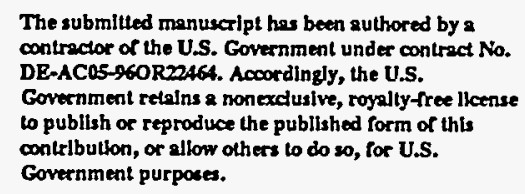

The submitled manuscripl hes been authored by a contractor of the U.S. Government under contract No. DE-AC05-960R20464. Acoordingly, the U.S. Govecnment retalns a nonexdusive, royal ty-free license to publlsh or reproduce the published form of this contrlbution, or allow others to do so, for U.S. Government purposes.

\section{DISCLAIMER}

\begin{abstract}
This report was prepared as an account of work sponsored by an agency of the United States Government. Neither the United States Government nor any agency thereof, nor any of their employees, makes any warranty, express or implied, or assumes any legal liability or responsibility for the accuracy, completeness, or usefulness of any information, apparatus, product, or process disclosed, or represents that its use would not infringe privately owned rights. Reference herein to any specific commercial product, process, or service by trade name, trademark, manufacturer, or otherwise does not necessarily constitute or imply its endorsement, recommendation, or favoring by the United States Government or any agency thereof. The views and opinions of authors expressed herein do not necessarily state or reflect those of the United States Government or any agency thereof.
\end{abstract}

Managed by Lockheed Martin Energy Research Corp. under contract DE-AC05-960R22464 with the U.S. Department of Energy.

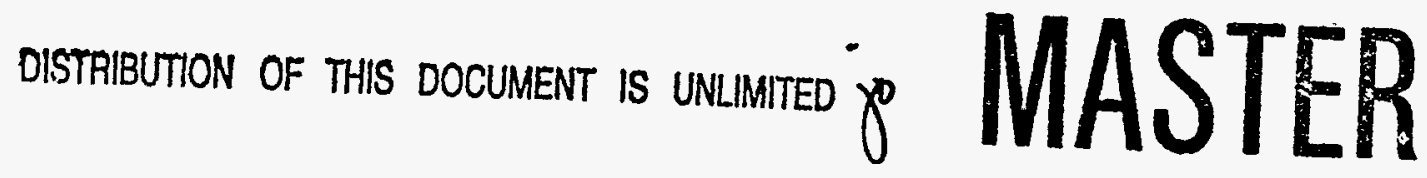




\section{J. A. Wang}

\section{USING ULTRASONIC MEASUREMENTS AND A TWO-PHASE COMPOSITE MODEL TO ASSESS RADIATION DAMAGE IN REACTOR PRESSURE VESSEL STEELS}

REFERENCE: Wang, J. A., "Using Ultrasonic Measurements and a Two-Phase Composite Model to Assess Radiation Damage in Reactor Pressure Vessel Steels," Nontraditional Methods of Sensing Stress, Strain, and Damage in Materials and Structures, ASTM STP 1318, George F. Lucas and David A. Stubbs, Eds., American Society of Testing and Materials, 1997

ABSTRACT: Ultrasonic methods used in the study of radiation damage and recovery in single crystals appear to also be useful for similar studies on polycrystalline alloys. Ultrasonic methods have demonstrated a sensitivity to radiation damage as affected by neutron fluence, irradiation temperature, large changes in composition, and possibly, as well, by neutron energy spectrum. On the microstructure defect evolution, only the residual defects created through the radiation event will contribute to the final macroscopic material property change. From a microstructure point, it is generally accepted that radiation hardening and embrittlement in metals are caused by clusters of vacancies, interstitial, and solute atoms that impede the motion of slip dislocations. Although vacancy-type defects are a major contributor to the material hardening, they also indicate the presence of other interstitial defects. Thus the total volume change of vacancy-type defects before and after irradiation can serve as a direct index to the final material property changes. The volume change of the vacancy-type defects can be determined by utilizing the two-phase composite model (matrix and void-type inclusion) to interpret wave velocities of baseline and irradiated specimens that are obtained from the ultrasonic wave experiment. This is a relatively economic and straightforward procedure. The correlation of the volume change of the vacancy-type defects with the existing destructive mechanical test results may play an important role in the future for the prediction of the radiation embrittlement and remaining plant lifetime, especially for the older plants on the verge of exhausting all the available mechanical test specimens loaded in the surveillance capsules. The above hypothesis was supported by the limited irradiated data analyzed and presented in this paper. The proposed ultrasonic methodology also has a potential application to assess creep damage in fossil power plants.

\footnotetext{
${ }^{1}$ Research Staff Member, Computational Physics and Engineering Division, Oak Ridge National Laboratory, Oak Ridge, Tennessee 37831.
} 
KEYWORDS: radiation embrittlement, ultrasonic, nondestructive, vacancy cluster, twophase composite, power reactor, pressure vessel steels

\section{INTRODUCTION}

It is well known that the fluxes of energetic neutrons and fission fragments in neutron chain reactors can displace significant numbers of atoms and thus alter physical and mechanical properties [1]. From a microstructure point, it is generally accepted that radiation hardening and embrittlement in metals are caused by clusters of vacancies, interstitial, and solute atoms that impede the motion of slip dislocations. The degree of embrittlement is conventionally correlated with fast neutrons or with total displacement per atom (dpa). The radiation embrittlement of reactor pressure vessel (RPV) materials depends on many different factors, primarily flux, fluence, neutron energy spectrum, irradiation temperature, preirradiation material history, and chemical compositions [2,3]. These factors must be considered to reliably predict the pressure vessel embrittlement and to ensure the safe operation of the reactor. Intensive studies have been and are being made to determine the parameters which seem to affect sensitivity to radiation embrittlement.

The aging and degradation of light-water-reactor (LWR) pressure vessels is of particular concern because of their relevance to plant safety and the magnitude of the expected irradiation embrittlement. The decrease of ductility in PRV materials is a direct consequence of irradiation hardening and embrittlement. Irradiation hardening can result in increase of yield and ultimate strengths, but decrease of ductility. Radiation damage of structural components would undoubtedly contribute to doubts against extending lifetime of power reactors. Changes in material properties are monitored by periodic testing of RPV surveillance specimens. Thermal annealing of reactor vessels may require additional testing to ensure the effectiveness of such treatments. There are only limited surveillance specimens loaded in the irradiated capsules, especially, for some older plants which may already be on the verge of exhausting all the available surveillance specimens. Therefore, it is essential to make the best use of the limited surveillance materials. Hence, there is a considerable benefit to be able to conduct nondestructive tests on the same surveillance specimen repeatedly and still be able to characterize the material properties of the reactor pressure vessel. The combination of the ultrasonic techniques and analytical methods will allow the determination of material moduli, strength, and toughness, and will provide a nondestructive approach to characterizing the initial states of materials and their degradation or modification due to exposure to service environments.

Ultrasonic methods, used in the study of radiation damage and recovery in single crystals [4], appear to be useful for similar studies on polycrystalline alloys. Ultrasonic methods have demonstrated a sensitivity to radiation damage as affected by neutron fluence, irradiation temperature [5], large changes in composition, and possibly, as well, by neutron energy spectrum. Ultrasonic methods offer further advantage of helping to define the nature of recovery mechanisms due to a theoretical differing ultrasonic response behavior between interstitial and vacancy-type defects $[6,7]$. 


\section{RADIATION DAMAGE INDEX}

Irradiation effects, or damage, on the physical, thermal, chemical and mechanical properties of nuclear reactor materials with time and other factors can greatly influence the reactor design, operation, performance, and safety in engineering applications. The change in these properties is attributed to the crystal (lattice) defects produced chiefly by neutron irradiation during reactor operation. The crystal defects induced by radiation on nuclear materials not only depend on the neutron flux, energy spectrum, irradiation time, and irradiation temperature, but also on the crystal structure, impurity atoms, alloying elements, heat treatment, etc. From the residual defects point, only the point defects that survive from the bulk recombination and in cascade recombination can contribute to the final mechanical property changes; the residual defects strongly depend on the material properties and irradiation environments, such as chemistry composition, heat treatment, irradiation temperature, and neutron spectrum.

From a microstructure point, vacancy clusters are thought to be responsible for more strengthening than are interstitial clusters at a given temperature and damage rate $[8,9]$. Vacancy clusters, such as dislocation loops, voids, and stacking fault tetrahedra, are strong obstacles to the movement of dislocations and, therefore, may determine the material hardening [10]. Currently, copper is considered as one of the primary contributors to the radiation embrittlement of the low alloy steel. Copper and other face center cubic (fcc) metals compositions are believed to stabilize vacancy-type defects in irradiation material [11-15].

From a mechanics point, the overall material properties, such as bulk modulus and shear modulus, depend on the properties of individual material components. If the volume or the property of the component undergo a large transformation, this component will definitely play a dominant role in the final overall material property change. The formation of the vacancy-type defect is such a candidate due to the dramatic change of material property in the matrix by forming a new phase with zero mass. Whereas for interstitial clusters, which generally have similar atomic numbers as that of a matrix, the sensing of change in material property may not be as great as that of vacancy clusters.

On the microstructure defect evolution, all the residual defects created through the radiation events will contribute to the final macroscopic material property changes. The residual defects in materials due to neutron-induced displacement damage are a function of neutron energy, neutron flux, and exposure temperature, as well as the material properties that determine how neutrons interact with atoms and how defects interact within the material. Vacancy cluster is a major contributor to the material hardening at higher temperatures and lower displacement rates, for example, at a commercial power reactor environment [9]. Thus from microstructure and mechanics points, vacancy-type defects not only can be considered as a major contributor to the material hardening, but also indicate the co-existence of other precipitates, such as copper. Furthermore, the total volume change of the vacancy-type defects may serve as a direct index to the final residual defects by taking into account the spectral effect, rate effect, and temperature effect, in addition to the material properties, implicitly. Therefore, the total volume change of the vacancy-type defects contained in the irradiated material may serve as a direct index to the radiation embrittlement. 
The volume change of the vacancy-type defect will serve as an important index to the radiation damage, and its correlation to the mechanical test results may play an important role in the future, especially for older plants on the verge of exhausting all the available mechanical test specimens loaded in the capsule of a RPV. The volume change of the vacancy-type defects can be determined by utilizing the two-phase composite model (matrix and void-type inclusion) to interpret wave velocities of baseline and irradiated specimens, obtained from the ultrasonic wave experiment. The detailed description of this proposed methodology is stated in the following sections.

\section{TWO-PHASE COMPOSITE MODEL}

\section{Background of Composites}

A composite material consists of a number of distinct homogeneous phases that form regions large enough to be regarded as continua and that usually bond together at the interface. Many natural and artificial materials are of this nature, such as some rocks (for example, sandstone), filled polymers, mortar, concrete, precipitate, porous and cracked media, etc. The development of mathematical models for composite materials is an extremely complicated and difficult task because of the large number of factors on which the behavior of the composite depends, such as phase factors [16-18], composition factors [19-21], and operational factors [22]. In order to develop a rigorous model for a composite, these factors must be under control, either by means of a particular model describing their influence or by suitable assumptions.

From a practical point of view, two kinds of information determine the properties of a composite material: the internal phase geometry [23] (i.e., the phase interface geometry) and the physical properties of the phase (i.e., the constitutive relation of these); the former is far more difficult to classify than the latter. Since the early 1960s, there has been much work on the elastic properties of heterogeneous materials [24-27]. Much of this work is of a fundamental nature and provides a sound basis for understanding and discussing the properties of aggregates and composites. Due to the complexity of composite materials, the exact analytical theory needs detailed modeling of the various factors, such as interfacial phenomena, debonding and contacting inclusions, and phase geometry, etc. The modeling of these factors is not always available, and then the effective bounds are usually too wide for practical application. For engineering purposes, a more practical and general model is desired. The modified direct method was developed to meet the above requirements [2 8 ]

\section{Modified Direct Method}

Two important assumptions are usually made when treating the macroscopic elastic properties of composite materials: statistical homogeneity and statistical isotropy [29]. For the problem to be workable, a necessary characteristic of a composite material is statistical homogeneity. This assumption essentially means that large enough subregions of the composite are statistically identical with the whole sample. The most frequently considered subregion of the multiphase medium is the two-phase composite in which one phase may 
be regarded as matrix in which arbitrarily shaped inclusions of other phases are embedded. The effective properties of a composite material, when their space variation is statistically homogeneous, define the relations between averages of field variables, such as stress and strain. The evaluation of an average elastic modulus of a composite material or polycrystals is one of the classical problems in micromechanics. The pioneering works have been done by Voigt and Reuss. The computation of effective properties in terms of the average is called the direct approach. In general, it requires determination of the appropriate fields in the phases as defined by the field equations, interface continuity conditions, and external homogeneous boundary conditions in order to compute the required average.

The modified direct method [28] is a scheme for the estimation of elastic moduli of composite materials and is based on micromechanical theory and classical elasticity. Using the statistical homogeneous assumption and the two-phase composite approach, one takes the average field of the composite. The spherical shape inclusion is the mean geometry of inclusions with a range of spectrum of arbitrary shapes, and is used in the analysis. In ref. 28 , comparison of modified direct method with experimental data and with other wellknown methods, such as Christensen-Lo, Kuster-Toksöz, and the self-consistent method, shows that the modified direct method provides a very good estimation of the elastic moduli in different kinds of problems, such as the soft and hard inclusion cases, porous materials, at various concentrations and/or various porosities.

Based on the theoretical derivations in the modified direct method for the composites, the effective moduli, $\bar{\mu}$ and $\bar{K}$, for the isotropic materials, can be written as

$$
\begin{aligned}
& \bar{\mu}=\mu+c_{2}\left(\mu^{*}-\mu\right) \bar{\alpha} \\
& \bar{K}=K+c_{2}\left(K^{*}-K\right) \bar{\beta}
\end{aligned}
$$

For small concentrations, $\bar{\alpha}$ and $\bar{\beta}$ can be written as

$$
\bar{\alpha}=\frac{(15 K+20 \mu) \mu}{(9 K+8 \mu) \mu+(6 K+12 \mu) \mu^{*}}, \quad \bar{\beta}=\frac{(4 \mu+3 K)}{4 \mu+3 K^{*}}
$$

where $\mu$ and $\mu^{*}$ are the shear moduli for the matrix and inclusion, respectively. $K$ and $K^{*}$ are the bulk moduli for the matrix and inclusion, respectively, and $c_{2}$ is the concentration of the inclusion.

For materials with a high concentration of inclusion or high porosity, the concept of an infinite matrix in the composite domain cannot be used. Instead, a finite subdomain containing the inclusion lumped and originating at a central position, maintaining the same volume ratio as in the original composite material, was used in the modified direct method. These subdomains are evenly distributed throughout the whole domain. The derived effective stiffnesses can be written as

$$
\begin{aligned}
& \bar{\mu}=\mu+c_{2}\left(\mu^{*}-\mu\right) \bar{\alpha}^{M} \\
& \bar{K}=K+c_{2}\left(K^{*}-K\right) \bar{\beta}
\end{aligned}
$$


where $\bar{\alpha}^{M}$ can be written as

$$
\bar{\alpha}^{M}=1+(1-R)(\bar{\alpha}-1)=\bar{\alpha}(1-R)+R
$$

and $R$ is defined as the modification factor, and can be written as

$$
R=\left(\frac{(1.38 K+0.88 \mu) c_{2}}{3 K+4 \mu}\right)^{2}\left(\frac{\bar{\alpha}}{\mu}\right)\left(\mu^{*}-\mu\right)
$$

\section{The Relation Between Acoustic Velocities and Elastic Modulus}

The elastic properties can be determined by measuring the acoustic velocities, $V_{P}$ (compression wave) and $V_{S}$ (shear wave), in a sample specimen. Once the wave speeds and density are known, then the elastic moduli can be determined directly from the following relations:

$$
K=\rho\left(V_{P}^{2}-\frac{4}{3} V_{S}^{2}\right), \mu=\rho V_{S}^{2}
$$

where $\rho$ is mass density. The Young's modulus $E$ and the Poisson's ratio $v$ can be determined as follows:

$$
E=\frac{9 K \mu}{3 K+\mu}, \quad \nu=\frac{3 K-2 \mu}{6 K+2 \mu}
$$

The experimental apparatus used to measure the wave speeds of the sample specimens is depicted in Fig. 1, which contains a block diagram of the experimental set-up. The system is composed of a pulsed oscillator, digital oscilloscope and Carver press, wave switch box, transmitter and receiver transducer, data acquisition system, mini computer, and plotter. The input signals are provided by a pulse generator for velocity determinations. A delay line incorporated in the oscilloscope is used for travel time measurements.

Interpretation of Radiation Damage by Ultrasonic Waves and Two-Phase Composite Model

The total volume change of vacancy-type defects before and after the irradiation can be used as a primary index to the radiation damage. Therefore, the overall radiation damage to the material can be estimated through the proper calibration of total volume change of vacancy-type defects. 


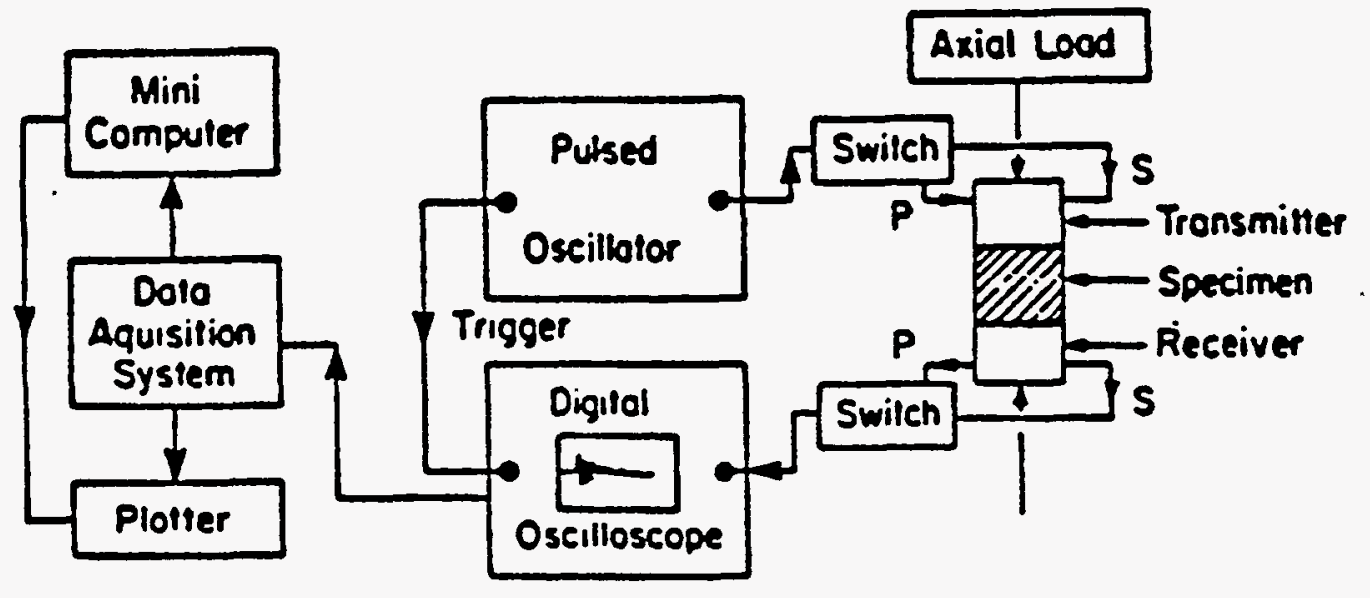

FIG. 1--Ultrasonic experimental set-up.

The original unirradiated sample can be defined as the matrix, and the post-irradiated sample can be defined as a two-phase composite, which includes the original matrix plus the void-type inclusions (the vacancy-type defects generated through neutron radiation event). Based on this two-phase composite model and applying Eqs 1-6 to analyze wave velocities for both unirradiated and irradiated samples obtained from the ultrasonic waves experiment, the quantity of the total volume change of vacancy-type defects before and after irradiation can be determined.

A preliminary analysis of Trantow's ultrasonic data on 304 stainless steel [30], which incorporated a two-phases composite modeling, shows that the estimated total volume change of vacancy-type defects increased with increasing neutron fluence, as illustrated in Fig. 2. These analytical ultrasonic test results demonstrated a very similar trend as that obtained from the destructive mechanical test results; namely, increased embrittlement damage with increased fluence. This trend demonstrates the potential of using the proposed new parameter (total volume change of vacancy-type defects) for evaluating neutron damage in typical low-alloy RPV steels.

Furthermore, Fig. 2 clearly indicates that the defects generation rate of vacancy-type defects are strongly dose dependent, that is at relative low dose the damage production rate of vacancy-type defects is small. This stage can be analogous to the incubation or nucleation stage, where after a certain threshold dose, the defects production start to take off, in analogy to the growth stage.

Currently, dpa is used as a measure of the potential to create point defects. It is not necessarily equal to, or even proportional to, the actual number of residual point defects. Clearly, a complete and accurate description of microstructural development during irradiation has not been attempted and seems to be beyond the present state-of-the-art. However, a better correlation parameter for radiation embrittlement can be expected through the upgraded dpa that incorporates the concepts of residual defects. 


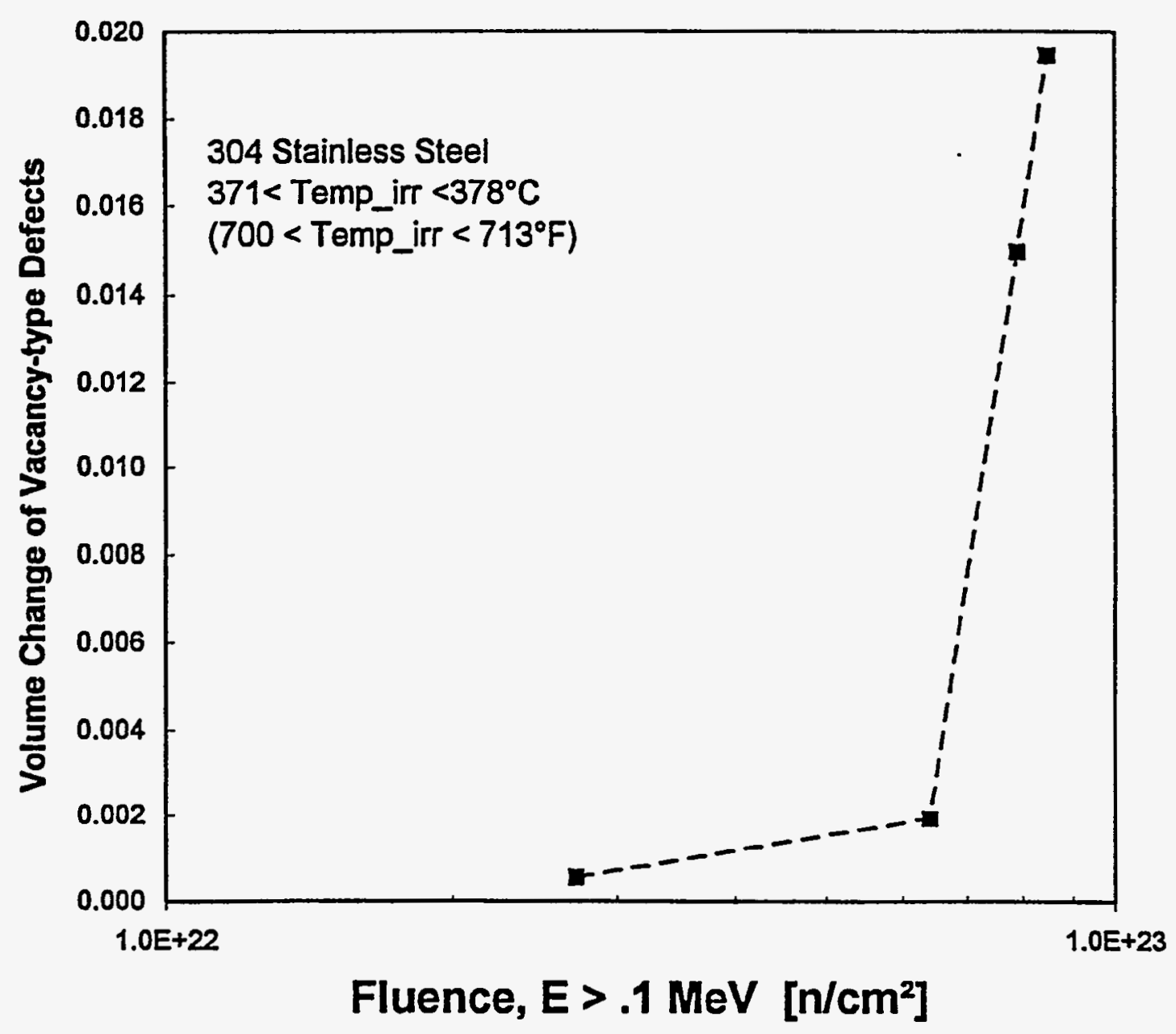

FIG. 2--Plot of total volume change of vacancy-type defects vs fluence.

\section{CONCLUSION ·}

This paper has demonstrated the feasibility of using ultrasonic nondestructive measurements and analytical techniques to characterize the primary parameter of radiation damage. The correlation of the analytical ultrasonic test results (volume change of the vacancy-type defects) with the existing destructive mechanical test results may play an important role in the future for the prediction of the radiation embrittlement and remaining plant lifetime. Moreover, the linear and nonlinear characteristics of the ultrasonic testing techniques also have potential applications to assess creep damage in fossil power plants and fatigue damage in structural components, and to estimate damage of structural members in components of the nation's infrastructures during service (such as bridges). 
Furthermore, the new integrated analytical/experimental ultrasonic testing technique will enable in-situ monitoring of material property changes during service. Then it will be possible to carry out inexpensive, faster, and more comprehensive assessments of both material quality and structural integrity.

\section{REFERENCES}

[1] Mansur, L. K., "Mechanisms and Kinetics of Radiation Effects in Metals and Alloys," Kinetics of Nonhomogeneous Processes, ed. by Gordon R. Freeman, 1987.

[2] Odette, G. R., Lombrozo, P. M., and Wullaert, R. A., "Relationship Between Irradiation Hardening and Embrittlement of Pressure Vessel Steels," Effects of Radiation on Materials, Twelfth International Symposium, ASTM STP 870, eds. Garner, F. A. and Perrin, J.S., American Society for Testing and Materials, Philadelphia, 1985, pp.840-860.

[3] Lucas, G. E., Odette, G. R., Lombrozo, P. M., and Sheckherd, J. W., "Effects of Composition, Microstructure, and Temperature on Irradiation Hardening of Pressure Vessel Steels," Effects of Radiation on Materials, Twelfth International Symposium, ASTM STP 870, eds. Garner, A. and Perrin, J., American Society for Testing and Materials, Philadelphia, 1985, pp. 900-930.

[4] Thompson, D. O., and Pare, V. K., "Use of An elasticity in Investigating Radiation Damage and Diffusion of Point Defects," Physical Acoustic Principles and Methods, Volume III-Part A, Mason, W. P., ed., Academic Press, New York, 1966, P. 293.

[5] D. O. Hunter, "Ultrasonic Velocities and Critical-Angle-Method Changes in Irradiated A302-B and A542-B Steels, "BNWL-988, May 9, 1969.

[6] G. J. Dienes, " A Theoretical Estimate of the Effect of Radiation on the Elastic Constants of Simple Metals," Physical Review, Vol. 86-2, p. 228, April, 1952.

[7] G. J. Dienes, "Effect of Radiation on Elastic Constants," Physical Review, Vol. 87-4, P. 665, August, 1952.

[8] Spitznagel, J. A., and Venskytis, F. J., "The Annealing of Copper Vacancy Aggregates in Neutron Irradiated Ferritic Pressure Vessel Steels, TRANS ANS 1975, p.161.

[9] Stoller, R. E., "Modeling The Influence of Irradiation Temperature and Displacement Rate on hardening Due to Point Defect Clusters in Ferritic Steels, "Effects of Radiation on Materials: 16th International Symposium. ASTM STP 1175, Arvind S. Kumar, David S. Gelles, Randy K. Nanstad, and Edward A. Little, Editors, ASTM, 1993, pp.394-423. 
[10] Jung, P., "Relevance of the Displacement Damage Concept for the Evaluation of Radiation Effects in Metals," Radiation Effects and Defects in Solids, Vol. 113, 1990, pp. 109-118.

[11] Brenner, S. S., Wagner, R. and Spitznagel, J.A. "Field Ion Microscope Detection of Ultra-Fine Defects in Neutron-Irradiated Fe-34\% Cu Alloy", Metallurgical Transactions, Vol. 9A, 1978, pp. 1761.

[12] Smitd, F.A. and Sprague, J.A. "Property Changes Resulting from Impurity-Defect Interactions in Iron and Pressure Vessel Steel Alloys," Effects of Radiation on Substructure and Mechanical Properties of Metals and Alloys, ASTM STP 529, 78, (1973).

[13] English, C. A., "Recoil Effects in Radiation Damage," Radiation Effects and Defects in Solids, Vol. 113, 1990, pp. 15-28.

[14] Simons, R. L., "Damage Rate and Spectrum Effects in Ferritic Steel NDTT Data," Influence of Radiation on Material Properties: 13th International Symposium (Part II). ASTM STP 956, F.A. Garner, C. H. Henager, Jr., and N. Igata, Eds., American Society for testing and Materials, Philadelphia, 1987, pp. 535-551.

[15] Odette, G. R., and Sheeks, C. K. ,"A model for Displacement Cascade-Induced Microvoid and Precipitator Formation in Dilute Iron-Copper Alloys," Phase Stability During Irradiation, J. R. Holland, L. K. Mansur, and D. I. Potter, Eds., Metallurgical Society of the American Institute of Mining, Metallurgical and Petroleum Engineers, (1982), p. 415.

[16] Bland, D.R. (1960). The Theory of Linear Viscoelasticity. Pergamon Press, Oxford, London.

[17] Tobolsky, A.V. (1956). "Stress Relaxation of the Viscoelastic Properties of Polymers." Journal of Applied Physics, Vol. 27, pp. 673-685.

[18] Timoshenko, S., and Goodier, J.N. (1969). Theory of Elasticity, Mcgraw-Hill.

[19] Hashin, Z. (1970). "Mechanics of Composite Materials." Proceedings, 5th Symposium. Naval Structure Mechanics.

[20] Wu, I.N., and Sendeckyj, G.P. (1974). "Multiple Circular Inclusion Problems in Plane Elastostatics." Journal of Applied Mechanics, Vol. 41, ser. E, pp. 215-221.

[21] Goree, J.G., and Wilson, H.B. (1967). "Axisymmetric Torsional Stresses in a Solid Containing Two Partially Bonded Rigid Spherical Inclusions." Journal of Applied Mechanics, Vol. 34, ser. E, pp. 313-320. 
[22] Theocaris, P.S., and Paipetis, S.A. (1978). "Indentation Studies in Aluminum-Filled Epoxies." Journal of Applied Polymer Science, Vol. 22, pp. 2245-2252.

[23] Papanicolaou, G.C., and Paipetis, S.A. (1978). "The Concept of Boundary Interphase in Composite." Colloid and Polymer Science, Vol. 256, pp. 625-630.

[24] Wilson, H.B., and Hashin, Z. (1962). "The Elastic Moduli of Heterogeneous Materials." Journal of Applied Mechanics, Vol. 29, pp. 143-150.

[25] Budiansky, B. (1965). "On the Elastic Moduli of Some Heterogeneous Materials." Journal of Mechanics Physics Solids, Vol. 13, pp. 223-227.

[26] Hill, R.A. (1965). "Self Consistent Mechanics of Composite Materials." Journal of Mechanics Physics Solids, Vol. 13, pp. 213-222.

[27] Walpole, L.J. (1969). "On the Overall Elastic Moduli of Composite Materials." Journal of Mechanics Physics Solids, Vol. 17, pp. 235-251.

[28] J. A. Wang, J. Lubliner, and P. J. M. Monteiro, "The Modified Direct Method for the Calculation of the Elastic Modulus of Composite Materials, "Proceedings of the First International Conference on Composites in Infrastructure, Eds. H. Saadatmanesh, and M. R. Ehsani, Tucson, Arizona, pp. 80-94, January 1996.

[29] Hashin, Z. (1964). "Theory of Mechanical Behavior of Heterogeneous Media." Applied Mechanics Reviews, Vol. 17, pp. 1-9.

[30] R. L. Trantow, "Ultrasonic Measurement of Elastic Properties in Irradiated 304 Stainless Steel," HEDL-TME-73-92, December 1973. 